\title{
RARE AND RADIATIVE $B$ MESON DECAYS FROM THE BABAR EXPERIMENT
}

\author{
JÖRG STELZER \\ Stanford Linear Accelerator Center, \\ 2575 Sand Hill Road, MS 61, \\ Menlo Park, CA 94025, USA \\ E-mail: stelzer@slac.stanford.edu
}

\begin{abstract}
Since its start in 1999 the BABAR experiment has collected a vast amount of data. Electron - positron collisions at the energy of the $\Upsilon(4 S)$ resonance have produced about 240 million coherent $B^{0} \bar{B}^{0}$ and $B^{+} B^{-}$pairs, opening the doors for exploration of rare $B$ meson decays. An overview of the electroweak penguin physics program of $B A B A R$ is given, the analysis of two specific decays is presented in detail.
\end{abstract}

\section{Introduction}

One of the primary scientific goals in high energy physics is to measure the parameters of the Standard Model (SM). The aim is to confirm the model that describes the basic relations between the particles and forces, or to find evidence for its incompleteness, often termed New Physics (NP). So far all experimentally acquired data point towards the validity of the SM. However, new generations of particle accelerators and detectors are producing ever larger datasets enabling us to search for NP in the regime where the SM predicts only small signals. These rare decays include penguin decays, CKM suppressed decays, and pure leptonic decays of B mesons. Most have branching fractions of $10^{-4}$ or less.

Penguin decays proceed through a $W q$ loop diagram with the emission of an additional particle to conserve energy and momentum. The twofold change of the quark flavor introduces an effective flavor changing neutral current into the SM, otherwise forbidden at tree level.

Penguin B decays with a suppressed tree contribution may exhibit direct $C P$ violation, the asymmetry in partial width between a decay and its $C P$ conjugate. Outside the $B$ meson system only weak evidence exists. Recent observation of direct $C P$ violation in the decay $B^{0} \rightarrow K^{+} \pi^{-}$has raised excitement within and outside the community. ${ }^{1}$

Ultimately the study of rare penguin decays, in particular theoretically

1

Contributed to Lake Louise Winter Institute: Fundamental Interactions,

20-26 Feb 2005, Lake Louise, Alberta, Canada

Work supported in part by Department of Energy contract DE-AC02-76SF00515 
clean leptonic final states, could lead to the discovery of NP. New constituents like the Higgs or SUSY particles could enter the penguin loop, leading to increased branching fractions or effects on other observables.

The interplay of weak and strong forces in weak $B$ decays is best described in the framework of the Operator Product Expansion (OPE) and the renormalization group. The OPE achieves separation of the longdistance contributions contained in the operator matrix element $Q_{i}$ and short-distant physics described by the Wilson coefficients $C_{i}$ (Eq. 1). The index $i$ runs over the contributing operators; in the literature $Q_{7} \ldots Q_{10}$ are usually associated with the various penguin decays.

$$
H_{\mathrm{eff}}=-\frac{G_{F}}{\sqrt{2}} \sum_{i} C_{i}\left(\mu, M_{W}\right) Q_{i}(\mu)
$$

While the coefficients can be calculated by well established field theoretical methods, it is - with few exceptions - the matrix elements that impose as the primary source of theoretical uncertainty. There are however a number of rare decays $\left(B \rightarrow X_{s} \gamma, B \rightarrow l^{+} l^{-}, B \rightarrow K \nu \bar{\nu}\right)$ ), where the matrix elements can be extracted from other measurements or calculated perturbatively. Those are of primary interest.

\section{The Rare Electroweak Physics Program at BABAR}

Depending on the gauge boson coupling to the loop one distinguishes between electroweak - that particle being a $W^{ \pm}, Z$, or $\gamma$, - and QCD - gluonic - penguin diagrams. The focus of this article will be the electroweak penguin decays with a photon or a lepton pair emerging from the loop.

These decays can be studied in an inclusive or an exclusive manner, each having its own set of advantages and disadvantages. The reconstruction of a particular channel, e.g. $B \rightarrow K^{*} \gamma$, gives a clean experimental signal. However, due to long-distance QCD effects, namely the exchange of soft gluons in the formation of the strange hadronic state, large theoretical uncertainties arise when relating the measured quantities to the parameters of the SM. On the contrary the theoretical description of an inclusive decay such as $B \rightarrow X_{s} \gamma$ is very clean, since it corresponds to the partonic weak decay $b \rightarrow s \gamma$. Only short distance physics is of relevance here and has been calculated up to next-to-leading order (NLO). The challenge here is on the experimenters side, since the methods for inclusively identifying a clean strange-hadronic samples are limited. In the following the search for the exclusive decay channel $B \rightarrow(\rho, \omega) \gamma$ and the inclusive measurement of the $B \rightarrow X_{s} l^{+} l^{-}$will be presented. 


\section{Search for $B \rightarrow(\rho, \omega) \gamma$}

Decays of type $b \rightarrow d \gamma$ are Cabibbo suppressed by a factor $\left|V_{t d} / V_{t s}\right|^{2}$ relative to $b \rightarrow s \gamma$. Their measurement provides a mean for the extraction of $\left|V_{t d} / V_{t s}\right|$ complimentary to the lifetime measurements of the $B_{d}$ and $B_{s}$ meson. The relation between the measured branching fractions and the SM parameters and QCD correction factors is given by Eq. 2 .

$$
\frac{\mathcal{B}(B \rightarrow(\rho, \omega) \gamma)}{\mathcal{B}\left(B \rightarrow K^{*} \gamma\right)}=\left|\frac{V_{t d}}{V_{t s}}\right|^{2}\left(\frac{m_{B}^{2}-m_{\rho}^{2}}{m_{B}^{2}-m_{K^{*}}^{2}}\right)^{3} \zeta^{2}(1+\Delta R)
$$

where $\zeta=\frac{\xi_{\perp}^{(\rho)(0)}}{\xi_{\perp}^{\left(K^{*}\right)(0)}}=0.85 \pm 0.1$ is the transition form factor ratio reflecting the $\mathrm{SU}(3)$ breaking in the $B \rightarrow K^{*}$ transition, and $\Delta R=0.1 \pm 0.1$ the weak annihilation correction ${ }^{2}$. The individual branching ratios relate as $\frac{1}{2} \mathcal{B}\left(B^{+} \rightarrow \rho^{+} \gamma\right)=\mathcal{B}\left(B^{0} \rightarrow \rho^{0} \gamma\right)=\mathcal{B}\left(B^{+} \rightarrow \omega \gamma\right)$, the combined result is calculated as $\mathcal{B}(B \rightarrow(\rho, \omega) \gamma)=\frac{1}{2}\left\{\mathcal{B}\left(B^{+} \rightarrow \rho^{+} \gamma\right)+\frac{\tau_{B^{+}}}{\tau_{B^{0}}}\left[\mathcal{B}\left(B^{0} \rightarrow \rho^{0} \gamma\right)+\right.\right.$ $\left.\left.\mathcal{B}\left(B^{+} \rightarrow \omega \gamma\right)\right]\right\}$ where $\frac{\tau_{B}}{\tau_{B^{0}}}$ is the $B$ meson lifetime ratio.

The signature of $B \rightarrow(\rho, \omega) \gamma$ events is a high energy photon, required to be between $1.5 \mathrm{GeV}$ and $3.5 \mathrm{GeV}$. The $\rho$ and $\omega$ are reconstructed in their major decay mode, $\rho^{+(0)} \rightarrow \pi^{+} \pi^{0(-)}$ and $\omega \rightarrow \pi^{+} \pi^{-} \pi^{0}$, and combined with the photon to form $B$ meson candidates. Backgrounds in this analysis include peaking $B$ decays such as $B \rightarrow K^{*} \gamma$, combinatoric $\Upsilon(4 S) \rightarrow B \bar{B}$ background, and continuum events $e^{+} e^{-} \rightarrow q \bar{q}$ with $q \in\{u, d, s, c\}$. Variables which are related to the rest of the event (ROE) are combined in a neural net $(\mathrm{NN})$, to separate continuum events from $B$ decays. Variables constrained by the kinematics of the event are put into a Fisher discriminant, to reduce backgrounds such as $B \rightarrow(\rho, \omega, \pi)\left(\pi^{0}, \eta\right)$. This includes e.g. the helicity angle of the daughters of the $\rho$ and $\omega$, which are transversely polarized $J=1$ states. The remaining peaking background are $B \rightarrow K^{*} \gamma$ events where the $K^{ \pm}$from $K^{*}$ has been misidentified as a $\pi^{ \pm}$, they exhibit a shift in $\Delta E$ due to the misidentification. The signal yield is extracted by simultaneously fitting the four variables $m_{\mathrm{ES}}=\sqrt{E_{\text {beam }}^{* 2}-\vec{p}_{B}^{* 2}}$, $\Delta E=E_{B}^{*}-E_{\text {beam }}^{*}$, the NN output, and the Fisher discriminant, using probability distributions derived from Monte Carlo events.

The analysis was performed on a data sample of $191 \mathrm{fb}^{-1}$. Figure 1 shows the result of the fit for the combined modes $B \rightarrow(\rho, \omega) \gamma$. The signal yield is $269_{-120-45}^{+126+40}$ events, the significance $2.1 \sigma$. BABAR published an upper limit for the branching fraction $\mathcal{B}(B \rightarrow(\rho, \omega) \gamma)<1.2 \times 10^{-6}$ at $90 \%$ C.L..

Reaching the lower boundaries of some of the SM predictions ${ }^{2}$, this 


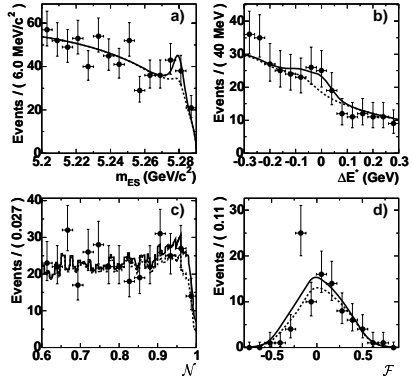

Figure 1. Fit of signal and background (dashed) PDF's simultaneously in $m_{\mathrm{ES}}, \Delta E$, NN, and Fisher on the combined $B \rightarrow(\rho, \omega) \gamma$ sample.

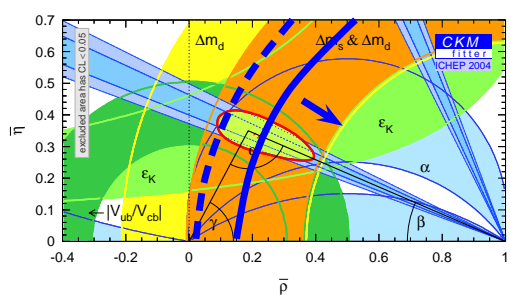

Figure 2. CKM constraint from $B \rightarrow$ $(\rho, \omega) \gamma . \quad[\zeta, \Delta R]=[0.85,0.1]-$ solid, $[0.75,0.0]$ - dashed.

result puts pressure on the calculation tools and models. BABAR sets an upper limit $\left|\frac{V_{t d}}{V_{t s}}\right|<0.19$ at $90 \%$ C.L., putting new constraints on the apex of the unitary triangle. However, within the errors of the $\zeta$ and $\Delta R$ it is consistent with the current CKM fit (Fig. 2).

\section{Semi-inclusive measurement of $B \rightarrow X_{s} l^{+} l^{-}$}

The decay $B \rightarrow X_{s} l^{+} l^{-}$receives short distance contributions from electromagnetic and $Z$ penguin diagrams as well as $W$ box diagrams. Long distance contributions are from the resonant process $B \rightarrow X_{s} J / \psi^{\left({ }^{\prime}\right)} \rightarrow X_{s} l^{+} l^{-}$ which can be eliminated by suitable cuts in the invariant lepton mass spectrum. While the branching fraction $\mathcal{B}\left(B \rightarrow X_{s} \gamma\right)$ depends on the magnitude of $C_{7}^{\text {eff }}$, its combination with various distributions of $B \rightarrow X_{s} l^{+} l^{-}$can be used to extract all short-distance physics from electroweak penguin diagrams $\left(C_{7}^{\text {eff }}, C_{9}\right.$, and $C_{10}$.) The BABAR analysis of $B \rightarrow X_{s} l^{+} l^{-}$is performed in a semi-inclusive fashion. Ten $B$ decay modes with a $K^{ \pm}$or a $K_{S}^{0}$ and a combination of up to three pions are reconstructed. Assuming the same rates for $K_{L}^{0}$ as for $K_{S}^{0}$ this accounts for about $70 \%$ of the total branching fraction. The precise number depends on the fragmentation model used and is the source of a large systematic uncertainty in the final result. After passing a $J / \psi$ and $\psi^{\prime}$ veto, lepton pairs with a minimum mass of $200 \mathrm{MeV} / c^{2}$ are combined with the strange hadronic system to form $B$ meson candidates. Several measures to suppress combinatoric background are taken. Among these the event shape variables $R_{2}$ and $\cos \theta_{\text {thrust }}$ and the variables $\Delta E, \Delta E^{R O E}$, and $m_{E S}^{R O E}$ prove to be most effective. The fit in the $m_{E S}$ distribution (Fig. 3) yields $40 \pm 10 \pm 2$ events. This results in a branching 

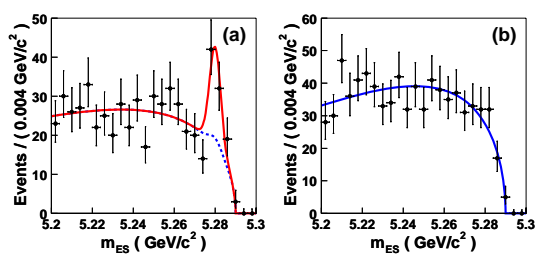

Figure 3. Fit to $m_{E S}$ for (a) $B \rightarrow X_{s} l^{+} l^{-}$ $(l=e, \mu)$ and (b) $B \rightarrow X_{s} e^{ \pm} \mu^{\mp}$ (lepton flavor violating).
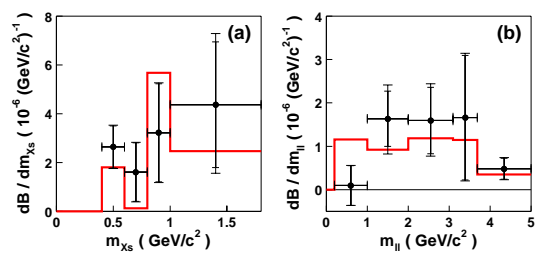

Figure 4. Differential branching fraction as a function of the (a) $X_{s}$ mass and (b) $l^{+} l^{-}$mass. Line - theory.

fraction measurement of $\mathcal{B}\left(B \rightarrow X_{s} l^{+} l^{-}\right)=(5.6 \pm .1 .5 \pm 0.6 \pm 1.1) \times 10^{-6}$, the errors being the statistic, systematic, and the previously mentioned model uncertainty. The differential branching fraction as a function of $m_{X_{s}}$ and $m_{l l}$ (Fig. 4) and the direct $C P$ asymmetry $A_{C P}$ are in good agreement with predictions. However, the precision of $A_{C P}$ is still about two orders of magnitude away from the theoretical uncertainty.

\section{Summary}

The BABAR collaboration has undertaken a number of analyzes in the electroweak penguin sector (Tab. 1). Many have reached a sensitivity at the level of the SM predictions; for those the tasks ahead are precision measurements of the branching fraction and first measurements of $C P$, forwardbackward, polarization, and isospin asymmetries. BABAR has not yet seen evidence of NP, but with the large data sample and sophisticated analysis techniques it has the best tools for a discovery at hand.

Table 1. Measurements published by the BABAR collaboration.

\begin{tabular}{|c|c|c|c|}
\hline & $B A B A R$ & Standard Model & \\
\hline \multirow{4}{*}{ 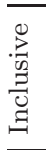 } & $B \rightarrow X_{s} \gamma$ & $\mathcal{B}=\left(3.88_{-0.56}^{+0.67}\right) \times 10^{-4}$ & $\mathcal{B}=\left(3.61_{-0.48}^{+0.36}\right) \times 10^{-4}$ \\
\hline & & $A_{C P}=(25 \pm 52) \times 10^{-3}$ & $A_{C P}=\left(4.2_{-1.4}^{+2.4}\right) \times 10^{-3}$ \\
\hline & $B \rightarrow X_{s} l^{+} l^{-}$ & $\mathcal{B}=(5.6 \pm 2.0) \times 10^{-6}$ & $\mathcal{B}=(4.2 \pm 0.7) \times 10^{-6}$ \\
\hline & & $A_{C P}=(220 \pm 260) \times 10^{-3}$ & $A_{C P}=(1.9 \pm 1.9) \times 10^{-3}$ \\
\hline \multirow{5}{*}{ 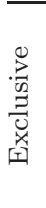 } & $B \rightarrow K^{*} \gamma$ & $\mathcal{B}=(4.06 \pm 0.26) \times 10^{-5}$ & $\mathcal{B}=(7 \pm 2) \times 10^{-5}$ \\
\hline & $B \rightarrow(\rho, \omega) \gamma$ & $\mathcal{B}<1.16 \times 10^{-6}$ & $\mathcal{B}=(1.38 \pm 0.42) \times 10^{-6}$ \\
\hline & $B \rightarrow K^{*} l^{+} l^{-}$ & $\mathcal{B}=(0.88 \pm 0.33) \times 10^{-6}$ & $\mathcal{B}=(1.19 \pm 0.39) \times 10^{-6}$ \\
\hline & $B \rightarrow K l^{+} l^{-}$ & $\mathcal{B}=(0.65 \pm 0.14) \times 10^{-6}$ & $\mathcal{B}=(0.35 \pm 0.12) \times 10^{-6}$ \\
\hline & \multicolumn{3}{|c|}{$\begin{array}{l}B^{+} \rightarrow K^{+} / \pi^{+} \nu \bar{\nu}, B^{0} \rightarrow l^{+} l^{-} / \gamma \gamma, B^{0} \rightarrow \phi \gamma \text { searches still } 1-10 \text { orders of magni- } \\
\text { tude away from SM predictions }\end{array}$} \\
\hline
\end{tabular}

\section{References}

1. B. Aubert et al. [BABAR Collaboration], Phys. Rev. Lett. 93 (2004) 131801

2. A. Ali, E. Lunghi and A. Y. Parkhomenko, Phys. Lett. B 595, 323 (2004) 